\title{
CASE-CONTROL STUDY OF SEROPOSITIVITY FOR CYSTICERCOSIS IN CUENCA, ECUADOR
}

\author{
KARYN A. GOODMAN, SUSAN A. BALLAGH, AND ARTURO CARPIO \\ Stanford University School of Medicine, Stanford, California; Health Sciences Research Institute, \\ University of Cuenca, Cuenca, Ecuador
}

\begin{abstract}
The prevalence of neurocysticercosis has been well documented in rural communities in Latin America using the enzyme-linked inmmunoelectrotransfer blot (EITB) assay. We studied the prevalence of neurocysticercosis in an urban, upper-middle class population in Cuenca, Ecuador. Family members of 34 index cases with parenchymal neurocysticercosis on a computed tomography (CT) scan and family members of 14 patients who had normal CT scans after a trauma or migraine were enrolled in the study. Serum was obtained from 226 individuals, 173 (72\%) from the case families and 67 (28\%) from the control families. Twelve percent of the case family members and $4 \%$ of the control family members were seropositive by the EITB assay. This was a statistically significant difference $(P$ $<0.05)$ when age and education were held constant by logistic regression. Seropositivity was not related to age. No neurologic symptom proved predictive of serostatus and the only demographic variable that correlated with seropositivity was increased crowding. Positive serology in index cases did correlate with CT findings as follows: $86 \%$ of patients with active lesions, $67 \%$ with transitional lesions, and only $41 \%$ of patients with inactive lesions were positive by the EITB assay. Eighteen percent of family members with a positive EITB test result had parenchymal lesions on a subsequent CT scan. This study demonstrates a high rate of seropositivity of cysticercosis among urban, middle to upper-middle class individuals in a region endemic for Taenia solium. Household contacts of patients with neurocysticercosis had a three-fold higher risk of positive serology for cysticercosis, in comparison with controls.
\end{abstract}

Neurocysticercosis is considered the most common parasitic disease of the central nervous system, with the highest prevalence in developing countries of Latin America, Africa, and Asia. In countries where it is endemic, neurocysticercosis may affect $2-4 \%$ of the population. ${ }^{1}$ In many Latin American countries, such as Ecuador, it is the major cause of late-onset epilepsy, accounting for more than $50 \%$ of such cases. ${ }^{2}$ However, neurocysticercosis is not just found in remote areas or developing countries. Due to immigration from areas where cysticercosis is endemic, this parasitic infection is increasingly prevalent in the United States. ${ }^{3}$

Humans acquire cysticercosis by ingesting eggs of Taenia solium, the pork tapeworm, in food or water contaminated by human feces of a tapeworm carrier. In humans, the $T$. solium larvae commonly migrate and develop into cysticerci in the central nervous system (CNS) (neurocysticercosis), but they may also be found in subcutaneous tissue, muscle, the eye, and in other parts of the body. ${ }^{4}$ The infection of the brain parenchyma by the larvae causes inflammation and creates cysts that can grow and cause mononuclear infiltrates, reactive astrogliosis, and cerebral edema, with possible displacement of neural structures. ${ }^{3}$ The signs and symptoms depend on the localization of the cysts and whether they are alive, dying, or dead and calcified. Epilepsy and intracranial hypertension are the most common sequelae. ${ }^{4}$

Much of the research on $T$. solium cysticercosis has focused on determining prevalence rates, assessing the accuracy of diagnostic methods, and comparing therapeutic regimens..$^{5-7}$ These investigations have established that neurocysticercosis has public health significance because of its medical and economic impact on both humans and livestock. Operational research is needed to define interventions to limit these parasites. In particular, identifying the seroprevalence of cysticercosis within household contacts may profile individuals at higher risk. Risk factors for neurocysticercosis demonstrated in studies from Mexico and Peru are 1) porcine husbandry, 2) an age of 20 years or older, 3) recollection of passing tapeworm proglottids (taeniasis), and 4) a family member with taeniasis. ${ }^{8-11}$ There are no data on the family members of patients with neurocysticercosis who are likely to be at higher risk of cysticercosis or taeniasis and may be an appropriate target population for preventive strategies.

The purpose of this case-control study was to determine the rate of seropositivity among family members of patients diagnosed with neurocysticercosis by computed tomography (CT) compared with family members of controls who had a negative CT scan. We used the enzyme-linked immunoelectrotransfer blot (EITB) assay to study the seroprevalence of cysticercosis among family members of index cases of neurocysticercosis and among controls in the city of Cuenca, Ecuador. We also surveyed participants about the occurrence of neurologic symptoms and past parasitic infections in an attempt to predict those at greatest risk for cysticercosis.

\section{MATERIALS AND METHODS}

Sample population. The case group consisted of family members of patients identified from three hospitals in Cuenca, Ecuador. Cuenca is the third largest city in Ecuador with a population of approximately 200,000 . It is located in the southern highlands of the Andes Mountains at an elevation of 2,534 meters. Cuenca has one of the highest standards of living in Ecuador and has a well-developed urban structure, three universities, six hospitals, and a medical school.

We selected patients from persons who had presented to the public Vicente Corral Moscoso Hospital, the Hospital del Seguro Social, or to the private Santa Inez Clinic and were diagnosed with parenchymal neurocysticercosis by CT between 1993 and 1996. A diagnosis of neurocysticercosis was made if one or more rounded, hypodense areas of variable size were present without contrast enhancement. ${ }^{6}$ Individuals with other severe medical illness (tuberculosis, stroke, cancer) were excluded. Only patients residing in the city of Cuenca were included. Controls were family members of 
TABLE 1

Distribution by age and sex of the studied sample, Cuenca, Ecuador, 1996

\begin{tabular}{|c|c|c|c|c|c|c|c|c|c|}
\hline \multirow[b]{2}{*}{ Age (years) } & \multicolumn{3}{|c|}{ All participants } & \multicolumn{3}{|c|}{ Cases } & \multicolumn{3}{|c|}{ Controls } \\
\hline & Men & Women & Total & Men & Women & Total & Men & Women & Tota \\
\hline $6-10$ & 12 & 15 & 27 & 7 & 13 & 20 & 5 & 2 & 7 \\
\hline $11-20$ & 30 & 50 & 80 & 23 & 41 & 64 & 7 & 9 & 16 \\
\hline $21-30$ & 15 & 29 & 44 & 13 & 17 & 30 & 2 & 12 & 14 \\
\hline $31-40$ & 19 & 28 & 47 & 15 & 22 & 37 & 4 & 6 & 10 \\
\hline $41-50$ & 15 & 18 & 33 & 12 & 16 & 28 & 3 & 2 & 5 \\
\hline $51-60$ & 8 & 11 & 19 & 5 & 6 & 11 & 3 & 5 & 8 \\
\hline $61-70$ & 7 & 7 & 14 & 4 & 5 & 9 & 3 & 2 & 5 \\
\hline$\geq 71$ & 2 & 8 & 10 & 2 & 6 & 8 & 0 & 2 & 2 \\
\hline Total & 108 & 166 & 274 & 81 & 126 & 207 & 27 & 40 & 67 \\
\hline
\end{tabular}

patients who had presented with trauma or migraine headaches to the three hospitals in the same period and had normal CT scan findings. The controls were matched for geographic and demographic characteristics. A total of 37 index cases diagnosed by CT with neurocysticercosis and 14 index controls who had undergone CT and had no evidence of neurocysticercosis were used to identify case and control family members who were eligible to participate in this study.

Data collection. Identified cases and controls and their families were contacted and informed consent was obtained under a protocol approved by the institutional review board of Stanford University. Home visits were made by the research team to survey family members and to draw blood for serologic analysis for anti-cysticercus antibodies. Unrelated individuals sleeping in the house were included as family members. A questionnaire provided information on household characteristics: ownership status, number of rooms, and number of bedrooms. Individual family members were queried about occupation, education, history of taeniasis or other parasitic infection, and neurologic symptoms including headache, dizziness, paralysis, and convulsions.

Laboratory studies. Venous blood samples were taken from all consenting members of case and control families six years of age or older. Serum was separated daily by centrifugation at the Hospital del Seguro Social in Cuenca, and stored in aliquots at $-5^{\circ} \mathrm{C}$. Sera was tested by an EITB assay (Immunetics, Inc., Cambridge, MA). Based on previous studies in Mexico, an EITB result was considered positive if there was a reaction of the serum sample with one or more of seven $T$. solium-specific glycoproteins. ${ }^{5}$ Prior studies have demonstrated this test to be highly sensitive (98\%) and specific $(100 \%) .{ }^{12}$ Results were expressed as either positive or negative. A positive EITB test result was expected with 1) intestinal taeniasis, 2) active neurocysticercosis, or 3) active cysticercosis outside the CNS. Results were provided to the participants free of charge along with recommendations and information concerning further follow-up including imaging by CT and therapeutic interventions when indicated. To validate the results of the serology in Ecuador, the immunoblot strips were sent to the Centers for Disease Control and Prevention (Atlanta, GA) for a second interpretation. Discrepant interpretations were read by a third physician to provide a definitive classification in less than $1 \%$ of the cases.

Computed tomography diagnosis of cysticercosis. All scans were performed on a GE CT Max (General Electric,
Fairfield, CT). Neurocysticercosis was classified by type of lesion under the criteria proposed by Carpio and others. ${ }^{13}$ Lesions seen on CT were classified as follows: 1) active cysticercosis: one or more rounded and hypodense areas of variable size seen without enhancement with contrast media; 2) transitional: one or more cystic areas, isodense with surrounding cerebral parenchyma, appearing only with administration of contrast media as nodular or annular forms; and 3) inactive cysticercosis: rounded, hypodense lesions, seen without contrast media, that had one or more areas of calcification.

Statistical analysis. Data entry and analysis was done using the SPSS (Chicago, IL) statistical package. Crude odds ratios were calculated to estimate the relative risk. Distribution of independent variables among case and control groups was analyzed by the chi-square test and $t$-test. Differences were considered statistically significant at $P<0.05$ (two-sided). Logistic regression was used to determine risk factors while holding confounding variables constant.

RESULTS

Sample characteristics. A total of 277 individuals in 51 households were enrolled in this study, of which three were index cases who had died by the study period, leaving a total of 274 participants. The number of persons per household ranged from three to 15 (mean $\pm \mathrm{SD}=7.2 \pm 3$ ). The distribution by age and sex is shown in Table 1. It was similar to the age and sex distribution of the province of Azuay, of which Cuenca is the capital. All of the households in this study had piped water, indoor plumbing, electricity and a mean \pm SD of $7.7 \pm 3$ rooms per house. More than threefourths $(76.5 \%)$ of the families owned their own home. Of the 274 participants, $207(75.5 \%)$ were in the case families and $67(24.5 \%)$ were in the control families. The two groups were similar in age and sex ratio (Table 1). The control families had significantly fewer household members per bedroom, 1.9 versus 1.5 . The case and control groups were similar for other socioeconomic markers including home ownership and level of education (Table 2).

Serology. Serum was obtained from 272 individuals, 226 of whom were family members, corresponding to 173 members of case families and 67 members of control families. Of the 226 samples tested by EITB for cysticercosis antibodies, $22(9.7 \%)$ were positive (Table 3$)$. The results of the immunoblot assay for each subgroup indicated that $11.6 \%$ 
TABLE 2

Demographic markers in case and control families

\begin{tabular}{lcc}
\hline & $\begin{array}{c}\text { Case } \\
\text { families }\end{array}$ & $\begin{array}{c}\text { Control } \\
\text { families }\end{array}$ \\
\hline$\%$ home owners & 78.5 & 70.1 \\
$\%$ completed high school* & 17.5 & 20.5 \\
Mean no. of persons per bedroom & $1.9 \dagger$ & 1.5 \\
\hline$*$ Among indiduals 25 years & &
\end{tabular}

$\dagger$ Statistically significant difference by $t$-test for equality of means $(P<0.05)$

(20 of 173) of the household contacts of patients with neurocysticercosis and $3.8 \%$ (2 of 53) of the control family members were seropositive. The relative risk for patients in case families was 3.0 (95\% confidence interval $=0.74-$ 12.7). This represented a statistically significant difference between the two groups at $P<0.05$ when age and education were held constant by logistic regression. There was no relationship between seropositivity and gender. Age had a bimodal distribution with peaks at $11-21$ and $31-40$ years of age. There were no seropositive individuals 61-70 years of age and only $0.4 \%$ of those $51-60$ years of age tested positive by EITB.

The remaining 46 serum specimens were collected from the index cases $(n=32)$ or controls $(n=14)$ used in identification of households selected for the study. All index controls had negative serology. Only 32 of the 37 initial index cases diagnosed with neurocysticercosis had a blood sample taken for EITB because three patients had died and two were unavailable for the blood draw. For these index cases, we correlated the results of the initial CT scan with the results from the EITB serologic assay. Only 15 of the 28 index cases for which we had EITB results were seropositive. When index case CT scans were categorized by type of lesion, six $(85.7 \%)$ of seven patients with an active lesion were seropositive, two $(66.7 \%)$ of the three patients with a transitional lesion on CT scan were seropositive, and only seven $(41.1 \%)$ of the 17 patients with inactive lesions were seropositive by the EITB assay (Table 4).

Clinical correlations of seropositivity. Among our study population, 180 individuals $(66 \%)$ reported in the screening questionnaire that they had provided stool for analysis for ova or parasites. Two of them (1.1\%) tested positive for $T$. solium. The outcome of the questionnaire for clinical neurologic signs and symptoms in persons seropositive for antibodies for $T$. solium by EITB and is shown in Table 5. Logistic regression showed that convulsions were significantly associated with EITB positivity for cysticercosis. Only one of 22 seropositive individuals actually reported seizures versus three of 202 seronegative family members. Headaches and dizziness were common in both groups.

Seventeen of the 22 individuals who were found to be seropositive by EITB subsequently obtained follow-up CT scans. Of these, three (18\%) showed neurocysticercosis.
TABLE 4

Correlation of enzyme-linked immunoelectrotransfer blot (EITB) assay results with computed tomography (CT) scan results among neurocysticercosis index cases and controls

\begin{tabular}{|c|c|c|c|c|}
\hline \multirow[b]{2}{*}{$\mathrm{CT}$ scan result } & \multicolumn{2}{|c|}{ EITB result } & \multirow[b]{2}{*}{ Total } & \multirow[b]{2}{*}{$\%$ positiv } \\
\hline & Negative & Positive & & \\
\hline Active & 1 & 6 & 7 & 85.7 \\
\hline Transitional & 1 & 2 & 3 & 66.7 \\
\hline Inactive & 10 & 7 & 17 & 41.1 \\
\hline Negative & 14 & 0 & 14 & 0 \\
\hline
\end{tabular}

Each had calcifications and one also had an active cyst. The individual with the active cyst had a history of intestinal taeniasis. However, none of these three family members with positive CT scans reported having convulsions. Only two reported headaches, and one reported dizziness. The remaining 14 individuals had normal CT results.

\section{DISCUSSION}

This study verified an increased seropositivity for antibodies to $T$. solium in family members of patients diagnosed with neurocysticercosis by CT. Family members of neurocysticercosis patients were three times more likely to be positive by EITB for cysticercosis than the general population. Increased seropositivity among family members of individuals with neurocysticercosis has been shown before in previous investigations in rural communities of Peru, Mexico, and Guatemala.,8,11 However, the earlier studies have focused on the prevalence of cysticercosis in rural areas and communities with lower socio-economic status. ${ }^{8-11}$ These studies reported a seroprevalence of human cysticercosis of $8 \%$ in Peru, ${ }^{4} 10.8 \%$ in Mexico, ${ }^{8}$ and from $10 \%$ to $17 \%$ in Guatemala. ${ }^{11}$ Our study demonstrates a high background rate of seropositivity for cysticercosis, $3.8 \%$, among an urban, middle to upper-middle class population, suggesting that socioeconomic status does not seem to mitigate against seropositivity in a region that is hyperendemic for $T$. solium taeniasis and cysticercosis. Unlike previous data from Mexico, ${ }^{9}$ showing a peak in subjects 46-55 years of age, and Guatemala, ${ }^{11}$ where there was a female predominance, there was no clear association between age or sex and seropositivity. All household contacts in this sample were equally susceptible to infection.

Previous studies have reported a poor correlation between Western blot and cerebral CT diagnosis of neurocysticercosis. ${ }^{13}$ There are several reasons for this. First, in prior studies, the EITB test was used as the standard to which the cerebral CT scan has been compared for sensitivity and specificity in the diagnosis of neurocysticercosis. In these studies, interpretation of CT scans was reported as being variant between radiologists. In our study, we found that interpretation of

TABLE 3

Enzyme-linked immunoelectrotransfer blot assay results

\begin{tabular}{lccc}
\hline & $\begin{array}{c}\text { Case } \\
\text { families }\end{array}$ & $\begin{array}{c}\text { Control } \\
\text { families }\end{array}$ & $\begin{array}{c}\text { Crude } \\
\text { odds ratio }\end{array}$ \\
\hline Seropositive & $11.6 \%(20 / 173)$ & $3.8 \%(2 / 53)$ & 3.06 \\
Seronegative & $88.4 \%(153 / 173)$ & $96.2 \%(51 / 53)$ & $0.74-12.7$ \\
\hline
\end{tabular}


TABLE 5

Comparison of neurologic symptoms among family members

\begin{tabular}{lccc}
\hline Reported symptoms & $\begin{array}{c}\text { Seronegative } \\
\text { individuals } \\
(\mathrm{n}=202)\end{array}$ & $\begin{array}{c}\text { Seropositive } \\
\text { individuals } \\
(\mathrm{n}=22)\end{array}$ \\
\hline None & $99(49.0 \%)$ & $11(50.0 \%)$ \\
Headache & $69(34.2 \%)$ & $5(22.7 \%)$ & $10.0 \%(11 / 110)$ \\
Dizziness & $19(9.4 \%)$ & $5(22.7 \%)$ & $(4.5 \%)$ \\
Convulsion a symptom
\end{tabular}

* Significant difference $(P<0.001)$.

${ }^{\dagger}$ Patients reported specific complaints such as tingling of extremities or other focal neurologic symptoms.

EITB strips was also discrepant between readers. Thus, the subjective reading of both tests can affect the overall correlation between the two. Second, it is not known how long detectable antibody titers persist in patients whose cysts have resolved. ${ }^{14}$ Garcia and others followed serology in 41 patients who were positive for neurocysticercosis by EITB. They found that only three $(7 \%)$ successfully treated individuals reverted to a negative serology within one year, ${ }^{15}$ suggesting that antibody titer persists despite treatment. This implies that serologic status was not indicative of the stage of the neurocysticercosis infection, assuming that a parallel can be drawn between the natural course of the disease and the effect of therapy on the immune response.

In our study, only $54 \%$ of the index cases diagnosed with neurocysticercosis by CT were seropositive by EITB. However, we found that the CT scan did predict serostatus when CT findings were classified by type of lesion. Our data reveal that six $(86 \%)$ of seven patients with active lesions on CT were seropositive. Fifty-nine percent of patients with inactive lesions became seronegative. Persisting antibody in the remaining $41 \%$ may represent continued parasitic infestation or recent death of the parasites. The seropositive individuals may have had gastrointestinal involvement of $T$. solium or cysticercus infections outside the CNS. This diminution in the rate of seropositivity among index cases with transitional and calcified lesions suggests that antibody titer will eventually disappear as the parasite dies and calcifies and is no longer stimulating an immune response. Longitudinal observation of serology during the course of treatment would also help to delineate whether the serology can represent a marker for active infection or therapeutic response. One limitation of this study is that we were unable to analyze stool for taeniasis. Simultaneous analysis of stool samples would detect coincident intestinal taeniasis and should be considered in future studies.

Prophylactic therapy of family members of neurocysticercosis patients has been proposed as a means of controlling the spread of $T$. solium eggs and subsequent cysticerci infection. ${ }^{16,17}$ While we did not have stool analysis for taeniasis among household members, two-thirds (180 individuals) reported in the questionnaire to have had a stool test for parasites. Of those, two (1.1\%) were positive for T. solium. This suggests a low rate of carriage of $T$. solium in these households. We did not collect samples from household help who did not sleep in the home. Many families had maids or cooks who could have introduced $T$. solium eggs into the household. Interestingly, the seropositive family member with an active cyst and multiple calcifications on a follow-up CT scan had a history of intestinal taeniasis, suggesting an autoinfection.

A three-fold increased rate of seropositivity of the family members of our index cases corresponded to previous results in Mexico, Peru, and Guatemala. ${ }^{8-11}$ The only predictive sociologic factor was increased crowding among affected families. Of the neurologic symptoms in Table 5, only the incidence of seizures showed a statistically significant difference. While this represented a three-fold higher rate of convulsions in seropositive individuals, the low frequency makes it a poor clinical indicator for public health screening purposes. However, the fact that greater than one of five (5 of 24) family members reporting a history of dizziness were seropositive for cysticercosis leads us to conclude that a history of dizziness in a high risk population should lower the threshold for performing serologic testing to rule out the presence of neurocysticercosis. Our follow-up of the 17 individuals who were found to be seropositive by EITB showed $18 \%$ with evidence of neurocysticercosis on CT. The three individuals that were positive by CT had only nonspecific neurologic symptoms. None reported a history of convulsions, making it a poor clinical indicator of infection. Due to the finding that one fifth of seropositive family members had neurocyticercosis by CT scan, we recommend a more detailed follow-up, including CT scans, of family members of neurocysticercosis patients, to evaluate this at-risk population for CNS disease. This would help to direct anti-cysticercal therapy. Since we did not have CT scan results from seronegative individuals, we cannot compare the rate of positive CT scans in these individuals with that of the seropositive group. Further investigation should focus on CT evaluation of both seronegative and seropositive family members to document reduced risk of CNS involvement in this group. This would validate the use of serology as a first-line screening test.

Acknowledgments: We thank the University of Cuenca medical students Marco Carrion, David Achig, and Geovany Abril for invaluable assistance in the data collection for this project. We also thank Dr. Victor C.W. Tsang (Department of Parasitology, Centers for Disease Control, Atlanta, GA) for helpful suggestions and assistance in the interpretation of EITB results, and Immunetics, Inc. for assistance in obtaining and transporting the EITB kits.

Financial support: Funding was provided by the Stanford Medical Student Traveling Scholars Program, a Stanford University Latin American Studies Travel Grant, and the Stanford University Institute for Research on Women and Gender.

Authors' addresses: Karyn A. Goodman, 329 Fulton Street, Palo 
Alto, CA 94301. Susan A. Ballagh, The Jones Institute, 601 Colley Avenue, Norfolk, VA 23507. Arturo Carpio, School of Medicine and Health Sciences Research Institute, University of Cuenca, PO Box 719, Cuenca, Ecuador.

\section{REFERENCES}

1. Vazquez V, Sotelo J, 1992. The course of seizures after treatment for cerebral cysticercosis. N Engl J Med 327: 696-701.

2. Del Brutto HO, Noboa CA, 1991. Late-onset epilepsy in Ecuador: aetiology and clinical features in 225 patients. $J$ Trop Geogr Neurol 1: 31-34.

3. Schantz PM, Moore AC, Munoz JL, Hartman BJ, Schaefer JA, Aron AM, Persaud D, Sarti E, Wilson M, Flisser A, 1992. Neurocysticercosis in an Orthodox Jewish community in New York City. N Engl J Med 327: 692-695.

4. Garcia HH, Gilman RH, Martinez M, Tsang VCW, Pilcher JB, Herrera G, Diaz F, Alvarado M, Miranda E, the Cysticercosis Working Group in Peru, 1993. Cysticercosis as a major cause of epilepsy in Peru. Lancet 341: 197-200.

5. Schantz PM, Sarti E, Plancarte A, Wilson M, Criales JL, Roberts J, Flisser A, 1994. Community-based epidemiological investigation of cysticercosis due to Taenia solium: comparison of serological screening tests and clinical findings in two populations in Mexico. Clin Infect Dis 18: 879-885.

6. Carpio A, Santillan F, Leon P, Flores C, Hauser WA, 1995. Is the course of neurocysticercosis modified by treatment with antihelminthic agents? Arch Intern Med 155: 1982-1988.

7. Cruz ME, Cruz I, Preux PM, Schantz P, Dumas M, 1995. Headache and cysticercosis in Ecuador, South America. Headache 35: 93-97.

8. Sarti E, Schantz PM, Plancarte A, Wilson M, Gutierrez IO, Lopez AS, Roberts J, Flisser A, 1992. Prevalence and risk factors for Taenia solium taeniasis and cysticercosis in humans and pigs in a village in Morelos, Mexico. Am J Trop Med Hyg 46: 677-685.

9. Sarti E, Schantz PM, Plancarte A, Wilson M, Gutierrez IO, Aguillera J, Roberts J, Flisser A, 1994. Epidemiological investigation of Taenia solium taeniasis and cysticercosis in a rural village of Michoacan State, Mexico. Trans $R$ Soc Trop Med Hyg 88: 49-52.

10. Garcia HH, Gilman RH, Tovar MA, Flores RJ, Tsang VCW, Diaz F, Torres P, Elba M, 1995. Factors associated with Taenia solium cysticercosis: analysis of nine hundred forty-six Peruvian neurologic patients. Am J Trop Med Hyg 52: 145148

11. Garcia-Noval J, Allan JC, Fletes C, Moreno E, de Mata F, Torrez-Alvarez R, Soto de Alfaro H, Yurrita P, Higueros-Morales H, Mencos F, Craig PS, 1996. Epidemiology of Taenia solium taeniasis and cysticercosis in two rural Guatemala communities. Am J Trop Med Hyg 55: 282-289.

12. Tsang VCW, Brand G, Boyer AE, 1989. Enzyme-linked immunoelectrotransferency blot assay and glycoprotein antigens for diagnosing human cysticercosis (Taenia solium). J Infect Dis 159: 50-59.

13. Carpio A, Placencia M, Santillan F, Escobar A, 1994. Proposal for a new classification of neurocysticercosis. Can J Neurol Sci 21: 43-47.

14. Garcia HH, Herrera F, Gilman RH, Catacora M, Tsang VCW, Pilcher JB, Diaz JF, Candy EJ, Miranda E, Naranjo J, 1994. Discrepancies between cerebral computed tomography and western blot in diagnosis of neurocysticercosis. Am J Trop Med Hyg 50: 152-157.

15. Garcia HH, Gilman RH, Catacora M, Verastegui M, Gonzalez AE, Tsang VCW, 1997. Serologic evolution of neurocysticercosis patients after antiparastic therapy. J Infect Dis 175: 486-489.

16. Drugs for Parasitic Infections, 1995. Med Lett Drugs Ther 37: 99-108.

17. Schantz PM, Cruz M, Sarti E, Pawlowski Z, 1993. Potential eradicability of taeniasis and cysticercosis. Bull Pan Am Health Organ 27: 397-403. 